\title{
3efferson.
}

JHN Journal

Volume 11 | Issue 1

Article 2

Winter 2016

\section{Continuous EEG Monitoring in Critical Care}

Andres Fernandez, MD

Department of Neurology, Thomas Jefferson University, andres.fernandez@jefferson.edu

Follow this and additional works at: https://jdc.jefferson.edu/jhnj

Let us know how access to this document benefits you

\section{Recommended Citation}

Fernandez, MD, Andres (2016) "Continuous EEG Monitoring in Critical Care," JHN Journal: Vol. 11 : Iss. 1 , Article 2. DOI: https://doi.org/10.29046/JHNJ.011.1.002

Available at: https://jdc.jefferson.edu/jhnj/vol11/iss1/2

This Article is brought to you for free and open access by the Jefferson Digital Commons. The Jefferson Digital Commons is a service of Thomas Jefferson University's Center for Teaching and Learning (CTL). The Commons is a showcase for Jefferson books and journals, peer-reviewed scholarly publications, unique historical collections from the University archives, and teaching tools. The Jefferson Digital Commons allows researchers and interested readers anywhere in the world to learn about and keep up to date with Jefferson scholarship. This article has been accepted for inclusion in JHN Journal by an authorized administrator of the Jefferson Digital Commons. For more information, please contact:

JeffersonDigitalCommons@jefferson.edu. 


\section{Continuous EEG Monitoring in Critical Care}

\author{
Andres Fernandez, MD ${ }^{1}$ \\ ${ }^{1}$ Department of Neurology, Thomas Jefferson University, Philadelphia, PA
}

\section{INTRODUCTION}

Continuous video-EEG monitoring (CEEG) has increasingly been used in the critical care population in large part due to the recognition that a wide variety of conditions are associated with the risk of developing seizures. As most seizures in this population are nonconvulsive, EEG provides the only reliable means to detect them and monitor response to their treatment. Below are common questions on the role of continuous EEG in the critical care patient followed by a brief overview.

\section{ARE SEIZURES COMMON IN CRITICAL CARE PATIENTS ?}

Seizures have been detected in $8-34 \%$ of critical care patients. ${ }^{1,2}$ The etiologies associated with a high risk of seizures include subarachnoid hemorrhage, intracerebral hemorrhage, traumatic brain injury, acute ischemic stroke, encephalitis, cardiac arrest (hypoxic-ischemic encephalopathy), sepsis, and pre-existing epilepsy. In a study of 570 patients with a variety of predominantly neurological etiologies undergoing CEEG for the detection of nonconvulsive seizures or unexplained impairment of consciousness, seizures were detected in $19 \%$, of which $92 \%$ were exclusively nonconvulsive. ${ }^{2}$ A more recent study of 625 adult inpatients undergoing cEEG (for $>18 \mathrm{~h}$ ) found an overall seizure frequency of $27 \% .{ }^{1}$ The frequency of nonconvulsive seizures is especially high after the control of convulsive status epilepticus. A prospective study of 164 patients with convulsive status epilepticus undergoing CEEG for a minimum of 24 hours found persistence of non-convulsive electrographic seizures after the control of convulsive status epilepticus in $48 \%$ of patients. ${ }^{3}$

While most studies have concentrated on critically ill neurological patients, cEEG often detects seizures in patients hospitalized in medical (MICU) and surgical (SICU) intensive care units. Oddo et.al. found a 10\% rate of electrographic seizures among $201 \mathrm{MICU}$ patients without known acute neurological injury undergoing cEEG (with purely electrographic seizures on $67 \%$ of cases); sepsis was a significant predictor of electrographic seizures and seizures were associated with poor outcome in this study. ${ }^{4}$ In another study of 105 patients without acute brain injury undergoing CEEG in the MICU and SICU, electrographic seizures were found in $11 \%$ of patients and they too were associated with worse functional outcome. ${ }^{5}$ Recently, Kurtz et.al. reported a $16 \%$ rate of nonconvulsive seizures among 154 SICU patients undergoing CEEG for altered mental status; nonconvulsive seizures were again associated with poor outcome. ${ }^{6}$

\section{DO SEIZURES HAVE AN IMPACT IN CRITICAL CARE PATIENTS ?}

Seizures induce physiological changes and have been associated with secondary brain injury. Vespa et.al. found an electrographic seizure rate of $6 \%$ in 46 patients with ischemic stroke and $28 \%$ in 63 patients with intraparenchymal hemorrhage $(\mathrm{ICH})$ undergoing cEEG (76\% had only electrographic seizures). In the ICH patients, seizures were associated with greater neurological deterioration and increase in midline shift on CT scans during the initial 72 hours after symptom onset. ${ }^{7}$ More recently, a study of 48 comatose subarachnoid hemorrhage patients undergoing multimodality monitoring including intracranial EEG recordings, showed a seizure rate of $38 \%$ for intracranial and $8 \%$ for surface seizures; intracranial seizures were associated with increases in heart rate, mean arterial pressure and respiratory rate reflecting a sympathetic response and with trends for increased intracranial and cerebral perfusion pressure. ${ }^{8}$ Seizures, and more specifically seizure burden, has recently been shown to independently contribute to neurological decline in a large prospective study in a pediatric critical care population. ${ }^{9}$ These associations suggest the potential impact of seizures in worsening the already fragile clinical state of the critical care patient

\section{WHAT ARE SOME COMMON USES OF CEEG MONITORING IN CRITICAL CARE PATIENTS?}

The most common indication for peforming cEEG in critical care patients is when there is a suspicion for seizures. As described above, there are many potential neurological and non-neurological etiologies that have a risk for seizures. Seizures might have a wide range of presentations. Critical care patients frequently have changes in the level of consciousness and/or altered mental status and the clinical exam alone is usually a poor marker for seizure detection in this setting, thus requiring cEEG for reliable diagnosis. When nonconvulsive seizures have clinical manifestations, they are typically subtle (e.g. non-overt rhythmic or repetitive movements, clonic, myoclonic, or tonic movements, gaze deviation, eyelid fluttering, etc) in contrast with the more overt rhythmic movements of the extremities seen in convulsive seizures. Critical care patients also frequently have paroxysmal events (e.g. motor or autonomic repetitive episodes) where an epileptic etiology is suspected and continuous EEG is a useful tool in establishing their potential epileptic origin. Given the subtle seizure semiology and the frequent occurrence of artifacts in the critical care setting, the video component of the EEG recording is of great importance.

In patients with status epilepticus, cEEG is key to assess the effectiveness of therapy. Its use in this setting is recommended by the recent neurocritical care guidelines for the treatment of status epilepticus. ${ }^{10}$ There are other indications for CEEG beyond seizure detection and seizure treatment monitoring including neurologic prognostication after cardiac arrest (as part of a multimodal approach); 11,12 there are also studies describing CEEG (quantitative EEG) use for ischemia detection in poor-grade subarachnoid hemorrhage patients, 13,14 and cEEG use for burst suppression monitoring to determine therapeutic endpoints during barbiturate coma. ${ }^{15}$ 
The 2012 Neurocritical Care Society guidelines are a valuable source for detailed recommendations on the indications for EEG monitoring in the critical care setting. These suggest strategies for evaluation and management of status epilepticus. In addition, the 2013 consensus statement on the use of EEG monitoring in critically ill patients from the Neurointensive Care Section of the European Society of Intensive Care Medicine is helpful. ${ }^{10,16}$

\section{WHAT IS THE TYPICAL DURA- TION OF CEEG MONITORING AND WHICH EEG FINDINGS ARE ASSOCIATED WITH HIGH RISK OF SEIZURES?}

There is no standard duration of cEEG monitoring. Factors such as the clinical state of the patient (e.g. comatose vs noncomatose), EEG findings (e.g. presence or absence of epileptiform abnormalities, periodic or rhythmic patterns), and underlying etiology may play a role in defining the individual duration of monitoring. Close communication between the intensivist and the neurophysiology teams can help in assessing monitoring duration for each patient. However, the literature contains data suggesting how EEG monitoring might be used.

In the retrospective study of 570 critical care patients undergoing $C E E G$, most seizures were detected within 24 hours of recording in non-comatose patients but longer monitoring periods were needed in comatose patients. Only $80 \%$ of the comatose patients had their first seizure within 24 hours of recording, with 13\% of the comatose patients needing $>48$ hours of recording to capture their first seizure.

The authors suggest that this data doesn't provide a guide for how long to monitor a patient but rather helps in the decision for cEEG discontinuation. ${ }^{2}$ In a recent study analyzing CEEG data from 625 inpatients monitored for varied, primarily neurological etiologies, the 72 hour risk of seizure could be determined based on the presence of epileptiform discharges.
The 72 hour risk of seizure decreases to < $5 \%$ in patients with no epileptiform activity over the first 2 hours of the recording and for patients with epileptiform activity but no seizures over the first 16 hours of the recording.

Only $4 \%$ of patients without epileptiform abnormalities had seizures, and $58 \%$ of patients who had seizures had their first seizure early in the recording $(<30 \mathrm{~min}$ of monitoring). ${ }^{1,17}$

The association of specific EEG patterns such as periodic or lateralized rhythmic patterns with seizures has been described in the literature. In a study of 67 comatose neuro-ICU patients undergoing prolonged CEEG monitoring (ten or more days), the presence of prolonged ( $\geq 5$ days), intermittent (1-5 days), or no recording of periodic epileptiform discharges (PED) was seen in $37 \%, 31 \%$, and $31 \%$ of patients, respectively. Prolonged PEDs were associated with the presence of electrographic seizures. ${ }^{18}$ Foreman et.al. reported data on 200 patients with generalized periodic discharges (GPDs) matched with 200 controls. Overall, $46 \%$ of patients with GPDs had a seizure (clinical or electrographic) during the hospital stay compared with $34 \%$ in controls. Nonconvulsive seizures and nonconvulsive status epilepticus were seen in $27 \%$ and $22 \%$ respectively in GPD patients compared with $8 \%$ and $7 \%$ in controls. ${ }^{19}$ In a study by Gaspard et.al. of 558 patients undergoing urgent EEG or $C E E G$, lateralized rhythmic delta activity (LRDA) was found in 27 subjects (5\%); lateralized periodic discharges (LPD) in 49 (9\%); focal nonrhythmic slowing in 136 (24\%); and no focal, periodic, or rhythmic patterns (labeled as controls) in 241 (43\%). Almost all subjects with LRDA, LPD or focal nonrhythmic slowing had an acute or remote cerebral injury. Almost two-thirds of patients with LRDA were stuporous or comatose.

A $63 \%$ rate of seizures during the acute illness (almost all electrographic) was seen for patients with LRDA, similar to the rate seen for subjects with LPDs (57\%), and higher than in nonrhythmic slowing and controls $(20 \%$ and $16 \%$ respectively). ${ }^{20}$
The presence of patterns that have an association with electrographic seizures in a given recording may warrant a longer recording than in ones where they are absent.

\section{IS THERE PRACTICE VARI- ABILITY ON THE USE OF CEEG IN CRITICAL CARE PATIENTS ?}

The variability in clinical practice in the use of continuous EEG in critical care patients has been recently highlighted on a survey of neurophysiologists and neurointensivists. One-hundred thirty seven physicians from 97 institutions completed the survey $164 \%$ institutional response rate). Almost all utilize cEEG for nonconvulsive seizure detection in patients with altered mental status after clinical seizures, intracerebral hemorrhage, traumatic brain injury, and cardiac arrest, and to characterize abnormal movements suspected to be seizures. There was variability in cEEG use for altered mental status in the setting of other etiologies such as tumors, ischemic strokes, central nervous system infections and metabolic encephalopathy where $>25 \%$ do not routinely perform $C E E G$ for these indications. The use for vasospasm detection after subarachnoid hemorrhage was low. Typical duration of monitoring was similar, with most reporting recordings lasting 24 or 48 hours (50\% and $29 \%$ respectively). Almost half of respondents reported an increase in CEEG use compared to the prior year. ${ }^{21}$

It is important to establish institutional protocols for indications and practice of critical care video-EEG monitoring. A team approach is ideal, which includes close collaboration between intensivists and the neurophysiology team. An upcoming consensus statement on critical care EEG currently under development from the American Clinical Neurophysiology Society will provide useful guidance in this process. 


\section{CONCLUSION}

In conclusion, continuous EEG plays an increasingly important role in the monitoring and treatment of critical care patients, both in the neurocritical care setting and in the general critical care population. Its role is expanding from the more typical use for seizure detection to include other uses such as prognostication of outcome and more generally for the neuromonitoring to aid management of the critically ill patient. There are still many unanswered questions and further research is needed. New insights into potential applications and overall significance in the care of the critically ill patient will be seen in years to come.

\section{REFERENCES}

1. Westover MB, Shafi MM, Bianchi MT, et al. The probability of seizures during EEG monitoring in critically ill adults. Clin Neurophysiol. 2014. (In-Press)

2. Claassen J, Mayer SA., Kowalski RG, Emerson RG, Hirsch LJ. Detection of electrographic seizures with continuous EEG monitoring in critically ill patients. Neurology. 2004;62(10):1743-1748

3. DeLorenzo RJ, Waterhouse EJ, Towne AR, et al. Persistent Nonconvulsive Status Epilepticus After the Control of Convulsive Status Epilepticus. Epilepsia. 1998;39(8):833-840.

4. Oddo M, Carrera E, Claassen J, Mayer SA, Hirsch LJ. Continuous electroencephalography in the medical intensive care unit. Crit Care Med. 2009;37(6):2051-2056

5. Kamel H, Betjemann JP, Navi BB, et al. Diagnostic yield of electroencephalography in the medical and surgical intensive care unit. Neurocrit Care. 2013;19(3):336-341.

6. Kurtz P, Gaspard N, Wahl AS, et al. Continuous electroencephalography in a surgical intensive care unit. Intensive Care Med. 2014;40(2):228-234.

7. Vespa PM, O'Phelan K, Shah M, et al. Acute seizures after intracerebral hemorrhage: A factor in progressive midline shift and outcome. Neurology. 2003;60(9):1441-1446.

8. Claassen J, Perotte A, Albers D, et al. Nonconvulsive seizures after subarachnoid hemorrhage: Multimodal detection and outcomes. Ann Neurol. 2013:74(1):53-64.

9. Payne ET, Zhao XY, Frndova H, et al. Seizure burden is independently associated with short term outcome in critically ill children. Brain. 2014:137(5):1429-1438.

10. Brophy GM, Bell R, Claassen J, et al. Guidelines for the evaluation and management of status epilepticus. Neurocrit Care. 2012;17:3-23.

11. Ben-Hamouda N, Taccone FS, Rossetti AO, Oddo M. Contemporary approach to neurologic prognostication of coma after cardiac arrest. Chest. 2014;146(5):1375-1386.
12. Sandroni C, Cariou A, Cavallaro F, et al. Prognostication in comatose survivors of cardiac arrest: An advisory statement from the European Resuscitation Council and the European Society of Intensive Care Medicine. Resuscitation. 2014;85(12):1779-1789.

13. Claassen J, Hirsch LJ, Kreiter KT, et al. Quantitative continuous EEG for detecting delayed cerebral ischemia in patients with poor-grade subarachnoid hemorrhage. Clin Neurophysiol. 2004;115(12):2699-2710.

14. Foreman B, Claassen J. Quantitative EEG for the detection of brain ischemia. Critical Care. 2012;16(2):216. (doi:10.1007/978-3-64225716-2).

15. Winer, JW., Rosenwasser $\mathrm{RH}$., Jimenez F. Electroencephalographic activity and serum and cerebrospinal fluid pentobarbital levels in determining the therapeutic end point during barbiturate coma. Neurosurgery. 1991;29(5):739-742.

16. Claassen J, Taccone FS, Horn P, Holtkamp M, Stocchetti N, Oddo M. Recommendations on the use of EEG monitoring in critically ill patients: consensus statement from the neurointensive care section of the ESICM. Intensive Care Med. 2013;39(8):1337-1351.

17. Dangayach N, Claassen J. Early epileptiform discharges and the yield of prolonged EEG monitoring. Clin Neurophysiol. 2014: (In-Press)

18. Ong C, Gilmore E, Claassen J, Foreman B, Mayer SA. Impact of prolonged periodic epileptiform discharges on coma prognosis. Neurocrit Care. 2012;17(1):39-44.

19. Foreman B, Claassen J, Abou Khaled K, et al Generalized periodic discharges in the critically ill: a case-control study of 200 patients. Neurology. 2012;79(19):1951-1960.

20. Gaspard N, Manganas L, Rampal N, Petroff OAC, Hirsch LJ. Similarity of lateralized rhythmic delta activity to periodic lateralized epileptiform discharges in critically ill patients. JAMA Neurol. 2013;70(10):1288-1295.

21. Gavvala J, Abend N, LaRoche S, et al. Continuous EEG monitoring: A survey of neurophysiologists and neurointensivists. Epilepsia. 2014;55(11):1864-1871. 